\title{
Dynamic analysis of wing deployment of high altitude balloon launched small size folding-wing UAV ${ }^{\mathbb{1}}$
}

\author{
Hangyue Zhang ${ }^{1,2}$, Yanchu Yang ${ }^{1,2}$ \\ ${ }^{1}$ University of Chinese Academy of Sciences, Beijing 100190, China \\ ${ }^{2}$ Academy of Opto-Electronics, Chinese Academy of Science, Beijing 100094, China
}

\begin{abstract}
The batch launch of small folding-wing UAVs by high altitude balloon at high altitude is a new technology in recent years. The wing deployment process at $20 \mathrm{~km}$ altitude is analyzed, and mathematical model of the wing delivery is established. Using the multibody dynamics analysis software ADAMS simulate the wing deployment process in order to verify the correctness of the model established. Then, the impact of the wing with final velocity on the fuselage is analyzed, and an elastic locking mechanism is designed to reduce the impact. The analysis process provides a certain reference for the task design of balloon-UAVs and the folding mechanism analysis.
\end{abstract}

\section{Introduction}

The near space of $20 \mathrm{~km}-100 \mathrm{~km}$ is between the traditional aeronautical area and the space flight area ${ }^{[1]}$. At present, the high-altitude balloon is a relatively mature near space vehicle. The release and launch aircraft by high altitude balloon is a technological means to develop and utilize near space resources and high altitude launch of small UAVs(Unmanned Aerial Vehicles) can make full use of the advantages of height. The mission profile of the UAVs launch by high altitude balloon is shown in Figure 1.

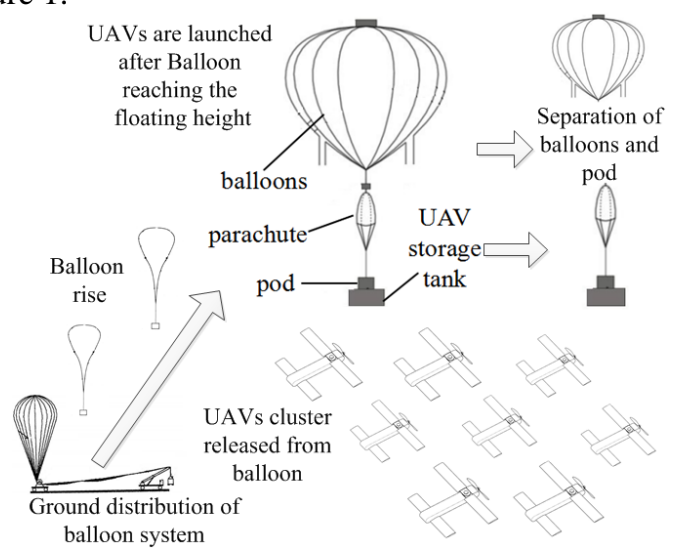

Figure 1. The mission profile of the UAVs cluster launched by high altitude balloon.

Folding wing UAV is the application of folding wing technology in UAV design process. Through the folding wings changes the dimension of the UAV, improving its storage, transportation, launch, recovery and aerodynamic performance ${ }^{[2]}$, which is beneficial to saving space and increasing the number of UAVs carried in one time. Such as the "Coyote" UAV (air launched, Fig. 2) and "vector hawk" UAV (submarine launched, figure 3), wing and tail both adopt a foldable design.

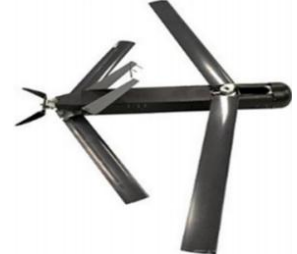

Figure 2. The "Coyote" UAV.

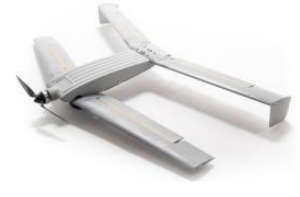

Figure 3. The "vector hawk" UAV.
The research on folding wing UAV is mostly focused on the study of aerodynamic influence of the front and rear wing layout, or the aeroelastic flutter analysis during the folding and expanding process ${ }^{[3-6]}$. The study of dynamic analysis and return vibration of the wing process is less ${ }^{[7]}$.The wing expansion performance is related to whether the UAV can fly normally after the launch. In this regard, many scholars have studied the process of wing expansion. Qin $\mathrm{Zhang}^{[7]}$ design the folding mechanism with spring as the power source, and the dynamic analysis and simulation are carried out. Wenqiang Zhen ${ }^{[8]}$ also designed the axial and spreading locking mechanism. For analysis method, most of researchers establish mathematical models of mechanism and combined with simulation software. Yushan Zhao ${ }^{[9]}$ set up the expansion process of the numerical solution of Lagrange equation. In addition, Jian $\mathrm{Ni}^{[10]}$ carried out the qualitative analysis of the reliability of the unfolding mechanism based on the FEMA principle. Bincai Qin ${ }^{[11]}$ used the sequence of two quadratic programming ( $S Q P$ )

*master student; research field:Technology of releasing and launching aircraft by aerostat

(1) $\rightarrow$ Supported by the National Defense Innovation Program of Chinese Academy of Science(CXJJ-16M206). 
method on folding and unfolding mechanism optimization. Considering that there is a certain terminal velocity after the deployment of the wing, the impact on the fuselage structure and the vibration of the wing will occur, which will affect the reliability of the wing deployment and the locking mechanism. In addition, the deployment of folding wing UAV at the stratospheric height, combined with the thin air of the stratosphere, and the smaller dynamic pressure of the wing during the deployment, is conducive to the rapid and reliable deployment of the wing.

\section{The establishment of the mathematical model and simulation}

UAV folding and unfolding mechanism is designed to carry out the wing reliably and reduce the impact on the fuselage. The UAV has a tandem wing layout. The area of the front and rear wings is roughly the same to increase the lift area. The airfoils select the Ritz airfoil, chord length is $8 \mathrm{~cm}$, single span $L_{A}=30 \mathrm{~cm}$. The UAV folding and unfolding sketches are shown in Figure 4, 5. The wing is powered by the torsion spring, and the wing is locked through the limit mechanism after the wing is fully unfolded. The wing angle, angular velocity, angular acceleration with time, and the end velocity of wing can be obtained. The final velocity dropped to 0 at the moment of collision with the fuselage, showing the impact on the structure of the body. Because the wing is a long straight beam with a ratio of more than 1, it will bring return vibration and propose a certain design requirement for the structure of the wing.

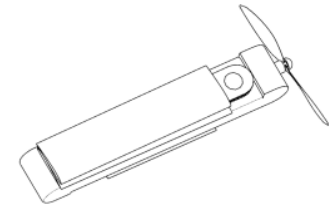

Figure 4. The unfolding shape of UAV.

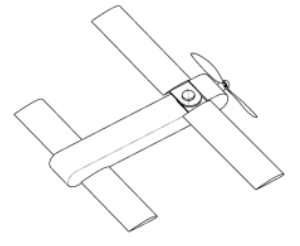

Figure 5. The folding shape of UAV.
The mathematical model shown in Figure 6 is established for the wing expansion process. $\theta$ is the unfolding angle $\left(0 \leq \theta \leq 90^{\circ}\right)$, small rotation angle $\Delta \theta$ with tiny time $\Delta t$ of the wing. $M_{\varphi}$ is a torsion spring driving torque. $F_{L}, F_{D}$ is the aerodynamic lift and drag. $M_{D}$ is air drag torque. $M_{\mu}$ is friction torque for rotating wing. $J$ is the moment of inertia around the shaft of the wing.

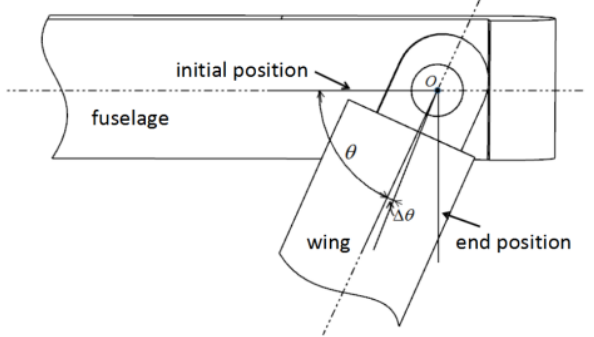

Figure 6. The mathematical model of wing expansion.
The motion equations of the wing are established as follows:

$$
M_{\varphi}-M_{D}-M_{\mu}=J \cdot \ddot{\theta}
$$

The initial condition $\theta_{(0)}=0, \dot{\theta}_{(0)}=0$, and set up SIMULINK module to get numerical solution. As figure 7, firstly, getting the relationship between acceleration with the angle of expansion, the angular acceleration at a certain time can be interpolated, and the angular velocity and angular displacement can get by integrating. And It is solved by ode 45 function with the time chosen as the independent variable.

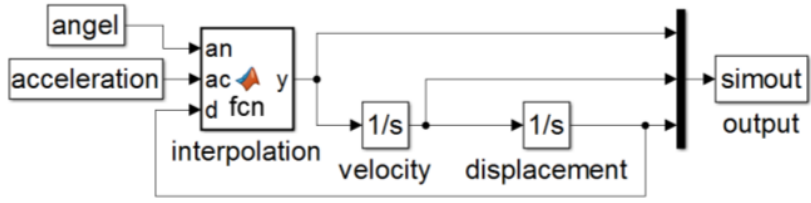

Figure 7. The SIMULINK module for numerical solution.

The aerodynamic drag and torque of the wing have different values in the unfolding process due to the different unfolding angles of the wing. The computational fluid dynamics (CFD) analysis is carried out on the wing with $4^{\circ}$ angle of attack under $20 \mathrm{~km}$ altitude and the environment flow velocity is $10 \mathrm{~m} / \mathrm{s}$. Using block and bonding technology and O-shaped grid to generate structured body fitted grid, and more than 10 grid nodes are arranged at the bottom of laminar flow. The steady state calculation is done, and the Simple algorithm is applied to get the aerodynamic parameters (lift coefficient $\overline{C L}$ and drag coefficient $\overline{C D}$ ) of the wing at different expansion angles, and the lift with drag of the wing are calculated. According to formula (2) the aerodynamic drag moment of wing can be derived.

$$
M_{D}(\theta)=0.5 \rho v^{2} \cdot S \cdot \bar{C} \bar{D}(\theta) \cdot L_{A} \cdot \sin (\theta) / 2
$$

Fitting out $\bar{C} \overline{\mathrm{L}} \sim \theta, \bar{C} \overline{\mathrm{D}} \sim \theta$ and $\mathrm{M}_{\mathrm{D}} \sim \theta$ curves as shown in Figure 8, 9.

The relationship between torsional spring torque and unfolding angle is expressed in formula (3).

$$
M_{\varphi}(\theta)=\frac{E d^{4}}{3667 D n} \theta
$$

The $E$ is elastic modulus of the twisted spring material. $d$ is the twisted spring line diameter. $D$ is the middle diameter of the torsion spring and the $n$ is the number of twisted reed rings .

The wing is subjected to ring frictional resistance moment at the joint of a wing shaft and it can be calculated according to formula (4).

$$
M_{\mu}(\theta)=2 \frac{2 \mu F_{L}(\theta)\left(R_{1}^{2}+R_{1} R_{2}+R_{2}^{2}\right)}{3\left(R_{1}+R_{2}\right)}
$$

$\mu$ is the coefficient of friction. $R_{1}, R_{2}$ is the large radius and small circle radius of the ring.

The formula (2-4) is brought into the formula (1) and the formula (5) is obtained, and the results shown in Figure 9 are obtained. 


$$
J \ddot{\theta}=\frac{E d^{4}}{3667 D n} \theta-2 \frac{2 \mu F_{L}(\theta)\left(R_{1}^{2}+R_{1} R_{2}+R_{2}^{2}\right)}{3\left(R_{1}+R_{2}\right)}-M_{D}(\theta)
$$

Now, the angle of the wing, angular velocity, angular acceleration variety with time, and the end velocity of the wing can be obtained as figure 10 displays.

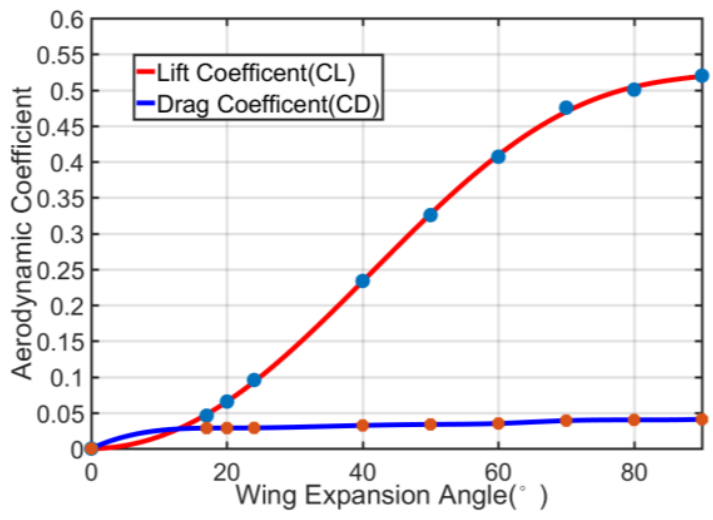

Figure 8. Curve of wing aerodynamic force with angle.

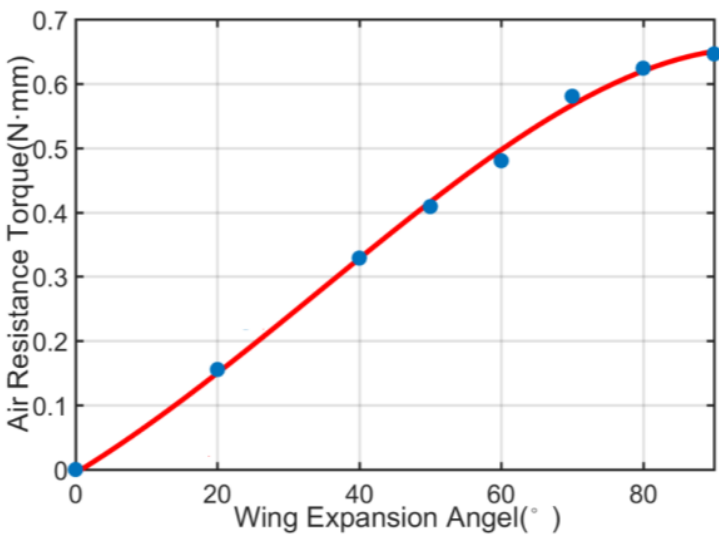

Figure 9. Curve of Wing aerodynamic drag torque with angle.

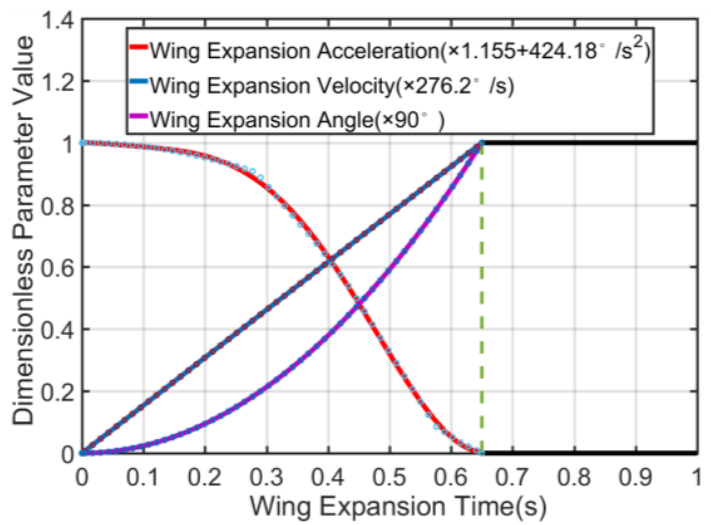

Figure 10. Kinematic curves obtained by solving mathematical models

From the result of the solution, it can be concluded that the wing process is completed in 0.65 seconds, the acceleration change little and angular velocity almost increase linearly. Finally, wing rotation is stopped at $90^{\circ}$.

The dynamics analysis software ADAMS is used for simulation, and the physical models of fuselage, wing, torsion spring and rotating shaft are established, giving the user defined driving force and driving torque of the wing center. The circular friction is realized by defining the indirect contact force of the component, and the nonlinear equivalent spring damping model based on the collision function algorithm is used to calculate. Contact force is expressed as

$$
F=K \delta^{e}+\operatorname{STEP}\left(\delta, 0,0, d_{\text {max }}, c_{\text {max }}\right) \cdot \dot{\delta}
$$

The $F$ is the normal contact force, $K$ is contact stiffness, permeability $\delta$, strength index $e$, and the step function STEP, $d_{\max }$ and $c_{\max }$ is the maximum penetration depth and the maximum damping value ${ }^{[}$. The simulation results show that the motion curve of the wing process is in agreement with the results of the mathematical model, and the correctness of the mathematical model is verified.

\section{Impact Dynamic analysis and design of locking mechanism}

The wing has a certain terminal velocity $(1.5 \mathrm{~m} / \mathrm{s})$ at the moment of fully unfolding, and it produces a shock to the fuselage, which is a transient dynamic problem. The direct explicit integration method of finite element analysis software ABAQUS is used to analyze the impact process, and the response curve of the impact force to the fuselage structure is obtained, as shown in Figure 11.

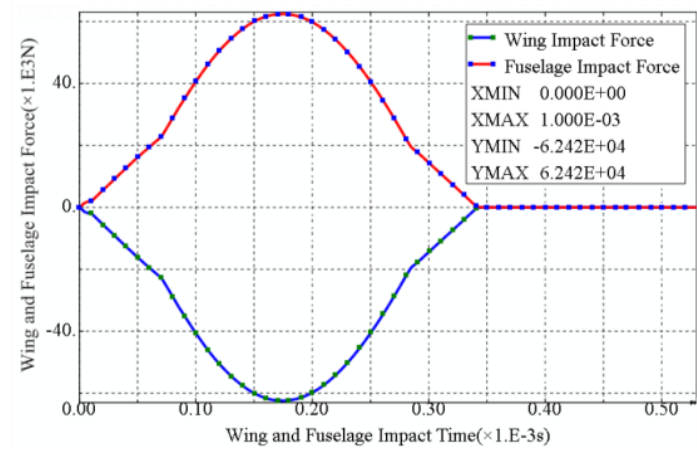

Figure 11. Instantaneous collision force curve of the wing and the fuselage.

From the analysis results, it can be found that the contact force of the collision process changes greatly, and the structure of the wing and the fuselage is certain to be destructive.

In order to reduce the hard impact of the wing on the fuselage, reference documentation [7] proposed the use of rubber cushion to shock absorption and give the simulation analysis. Considering the high throwing height and low temperature, the rubber property will become hardened and crisp, which is not suitable for high-altitude cushioning device. Inspired by the cushioning and damping characteristics of the elastic structure, and using friction to adsorb the kinetic energy, the locking mechanism shown in Figure 12 is designed. The locking mechanism has the elastic deformation of the structure under the impulse of the wing and ensures that the wing is always fixed during flight. Considering ABAQUS has powerful ability to solve nonlinear contact problems, it is used to analyze the locking mechanism dynamics either.

The dynamic analysis of the locking mechanism is carried out under the impact of the wing's terminal velocity with the obtained kinetic energy. The kinetic energy and internal energy variation curve of the elastic 


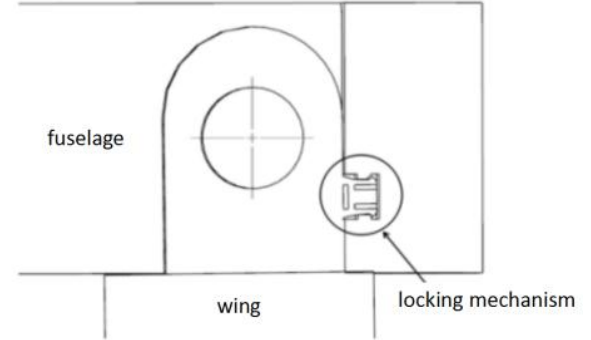

Figure 12. Elastic locking mechanism.

mechanism is shown in figures 13 and 14 . It can be seen that the kinetic energy of the wing is completely transformed into the internal energy of the collision, and the internal energy is mainly shown as the strain energy without elastic locking mechanism. And it can be drawn the wing is bounced back. Nevertheless, the kinetic energy of the wing is mainly transformed into friction dissipation with elastic locking mechanism, and the wing is locked tightly with kinetic energy reduced to 0 , which reduces the structural damage to the wing and the fuselage.

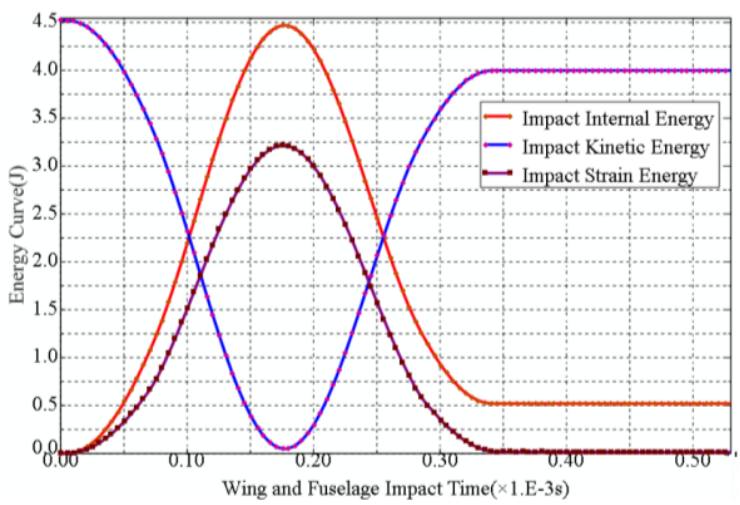

Figure 13. Energy change curves without locking mechanism.

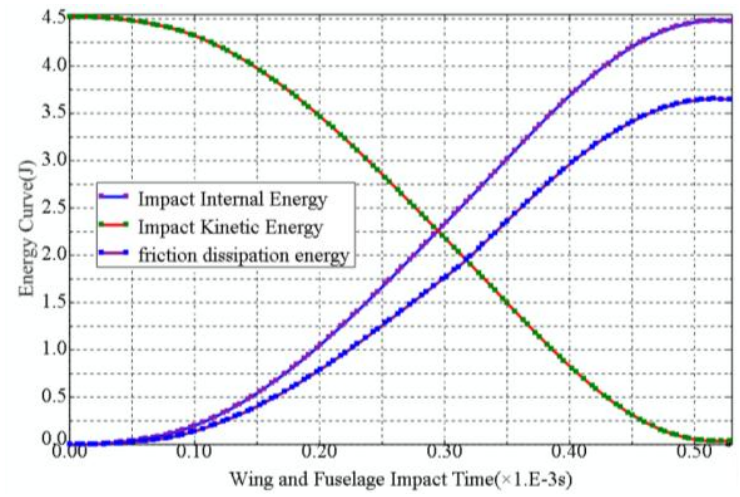

Figure 14. Energy change curves with locking mechanism.

\section{Summary}

The folding wing UAV is carried by a high altitude balloon to the stratosphere, and the wings unfold independently after the launch. The following several aspects have been analyzed.

(1) Combined with the design of the deployment mechanism, a mathematical model is established for the deployment process of the wing. The aerodynamic force and moment are calculated by the CFD tool. But only analyzed one wing at the moment, the tandem wing UAV has front and rear wings, should consider its interaction on aerodynamic analysis.

(2) The impact on the fuselage is analyzed at the end of the wing deployment speed. The impact will result in flutter of the wing and coupling with the structural dynamic behavior of the wing long straight beam. The impact on the structure is still needed to be further analyzed.

(3) In order to reduce the impact on the fuselage car, the locking mechanism is designed, and the elastic parts are added. Through simulation analysis, it can be concluded that the locking mechanism has a good effect on the reliable deployment and impact reduction of the wing.

The analysis results provide some reference for the design of the same kind of folding unfolding mechanism and the high altitude launch technology of UAV.

\section{References}

1. Gang Guo, Maoxing Shen. Present situation and development trend of military application in near space. Information Online. 2,16-21(2012).

2. Bo Liao, Changsheng Yuan, Yongze Li. The development status and key technology of the folding wing unmanned aerial vehicle. The Mechanical Design. 29,4(2012).

3. Chuanmei Bao, Changliang Liu, Ye Sun. Launching technology and development of UAV. Unmanned Aerial Vehicle. 2,56-60 (2012).

4. G.Q.Zhang, S.C.M.Yu. Unsteady Arodynamics of a Morphing Tandem-Wing Unmanned Aerial Vehicle. Journal of Aircraft. 49,5 (2012).

5. D.H.Lee, Terrence A.Weisshaar.Aeroelastic Studies on a Folding Wing Configuration. 46th AIAA Strucutres, Structural Dynamics \& Materials Conference.(2005).

6. Peter J.Attar, Deman Tang. Nonlinear Aeroelastic Study for Folding Wing Structures. AIAA Journal. 48,10(2010).

7. Qin Zhang, Hong Nie, Ming Zhang. Dynamic analysis of unmanned aerial vehicle folding wing expansion. Mechanical Design and Manufacturing Engineering. 44,2(2015).

8. Wenqiang Zhen, Yongqiang Ji. Dynamic simulation of folding wing deployment and locking process analysis. Information Technology. 2,62-65 (2016).

9. Yushan Zhao, Xudong Yu, Caixia Ma. The simulation of the folding wing expansion process. Missile, Arrow and Guidance Journal. 2,19-23(1997).

10. Jian Ni, Kai Lu, Duo Zhang. Reliability analysis of the launching mechanism of missile folding wing. Shanghai Spaceflight. 3,33-36 (2000).

11. Bincai Qin, Hua Li. The optimization design of small folding wing. Journal of Missiles and Guidance agencies. $29,4(2009)$.

12. Weidong Guo, Shouzhong Li, Lu Ma. ADAMS2013 application example tutorial. Beihang University press. (2008). 